\title{
Progress of Dynamical Nanomaterial Imaging Using Ultrashort Electron Pulses
}

\author{
Zhensheng Tao, He Zhang, Ramani K Raman, Tzong-Tu Han, Kiseok Chang, Martin Berz, and
} Chong-Yu Ruan

\section{Department of Physics and Astronomy, Michigan State University, East Lansing, MI 48824}

A driving force behind the development of ultrafast electron crystallography and microscopy is the recognition that there are a broad range of phase change materials that respond to photoexcitation in unusual pathways compared to those responding to applying pressure, heat, and chemical doping. Since the morphology of the nanomaterials play a role in determining their optical responses, the ability to resolve atomic structure and the transient dynamics that lead to various phase change states are crucial for these studies[1]. First, we will discuss the recent progress at MSU of using ultrafast electron crystallography and related methods to investigate nanomaterial assemblies[2]. We show that the dynamical responses of materials to a short-pulse (fs) laser excitation are multisphased, involving progressive transformations, from the initial femtosecond electronic excitation to ps and ns structural changes. In the study of the optically induced fragmentation of Ag nanocrystals excited at surface plasmon resonance, we find that the dominant dynamical feature in the prefragmentation stage is a defect-mediated instability growth, creating sub-nanocrystalline domains with hot surface and relatively cold core, based on diffraction refinements using a large supercell model[2], as shown in Fig. 1. From the defect density growth process, we suggest that the fragementaion is percolative, starting from valence modification as evidenced by creating topological defects, which are initially seeded in Ag through the strong nonlinear coupling between SPR and interband transition, causing chemical bonds to rupture. These defects will later percolates into larger stripes, thus causing the nanoparticles to fracture. Such creation and growth of topological defects, which can persist on the phonon timescales, could be common in nonequilibrium photoinduced structural phase transition. Further improving the temporal resolution to the phonon timescale (10-100fs) will allow more definitive traces of the initial nonlinear photoassisted electron-phonon coupling that seeds the reactions to be resolved.

To advance the spatiotemporal resolution, it is central to obtain a clearer understanding of the current limits in femtosecond electron pulse generation, including photoemission processes that define the initial statistical profile of the electron pulses, and the ensuing electron-electron interactions altering the spatotemporal profile of the electron pulses (space-charge effect), and the irreversible Boersch effect that ultimately limits the 6D compressible phase space of the electron bunches. To investigate the beam dynamics, we use an ultrafast electron point projection imaging approach[3], applicable to characterize these processes. The space-charge dynamics from a series of potential photoemitters, including thin gold film, silver nanocrystal, and carbon nanotube, were investigated. In the space-charge dominated regime, the nonlinear growth of the bunch width and power-law dependence (exponent $\sim 1 / 2$ ) on the particle number, up to $10^{6}$ electron/bunch are determined, as shown in Fig. 2a,b. A subtle near surface spacecharge effect is found to control the femtosecond laser induced photoemission processes, which influence the initial bunch profile and the ensuing space-charge dynamics. The clear dependence of the bunch evolution on the initial condition promises the ability to achieve control of the electron bunch phase space using femtosecond laser pulse shaping and RF high field that is properly tuned to achieve the optimum spatotemporal focusing. [4]. A new generation of nanoscale focusing ultrafast electron diffraction and imaging system using spatially and 
temporally shaped electron pulses for investigating nanomaterials on the single particle/domain site level will be presented (Fig.2c).
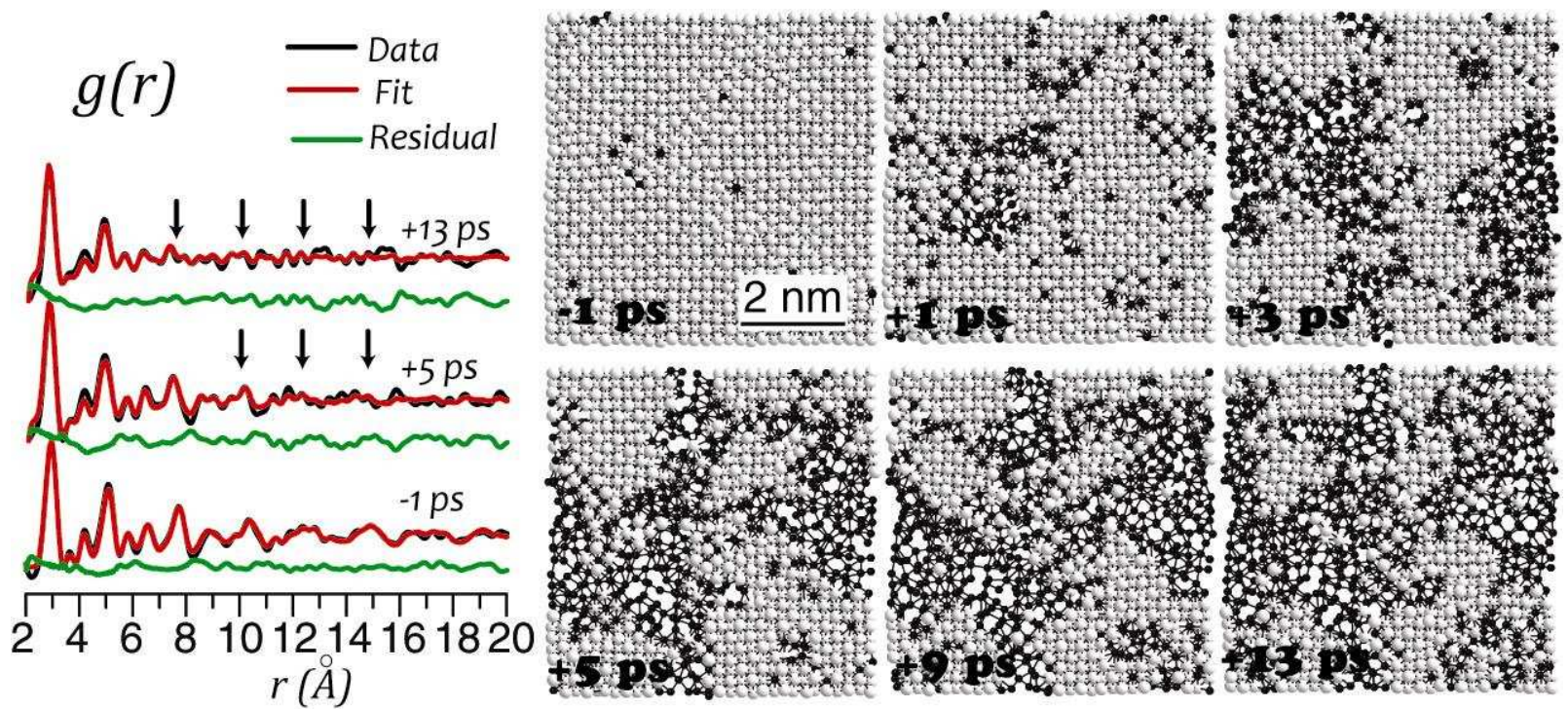

FIG.1. Investigation of the photoinduced fragmentation of $40 \mathrm{~nm} \mathrm{Ag}$ nanoparticles using ultrafast electron crystallography. The atomic structures (right), represented as a cut across a $15 \times 15 \times 15$ supercell model, are obtained by refining the diffraction data (left) using a reverse Monte Carlo method.
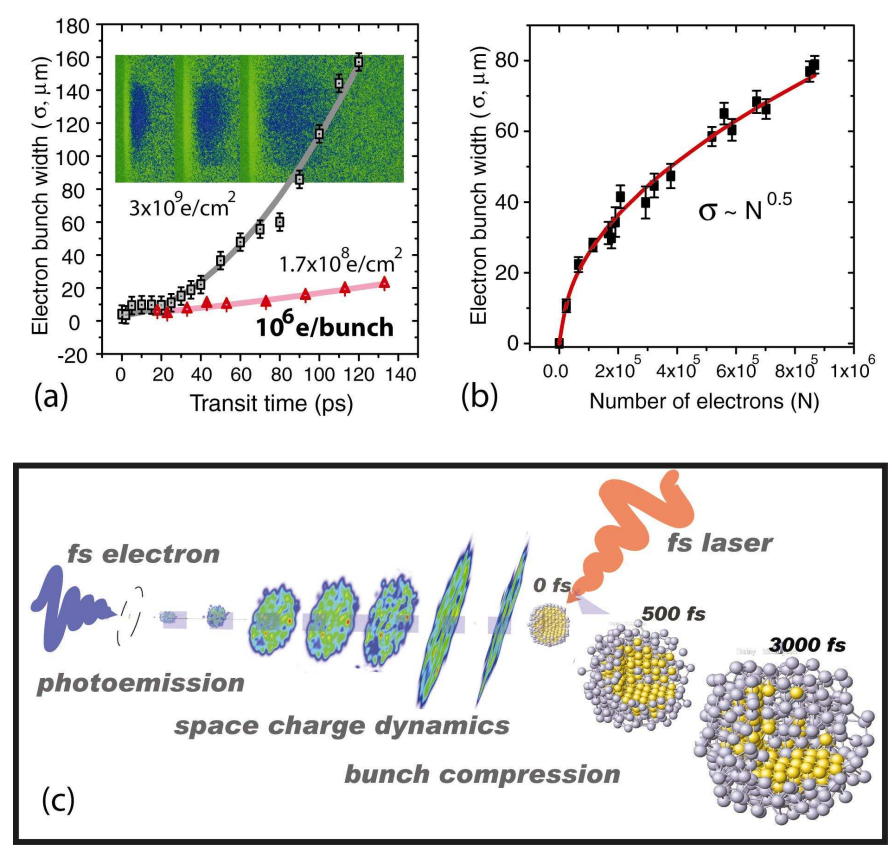

FIG.2. (a) The space charge dynamics following femtosecond photoemission determined using ultrafast electron point projection imaging method. The nonlinear growth of the electron bunch width $(\sigma)$ is evident as the density of the electron increases. (b) $\sigma$ as a function of number of electron/bunch (N), showing $\sigma \propto \sqrt{N}$ relationship. Note $\sigma$ is determined at transit time:100ps. (c) Concept of singleparticle electron crystallography using spatiotemporally focused electron pulses. The bunch profiles are obtained by numerical simulation of space-charge dynamics following photoemission. The bunch compression is achieved using a 1 $\mathrm{GHz}$ RF buncher and a magnetic lens.

References

[1] R.K. Raman, Y. Murooka, C-Y. Ruan, T.Yang, S. Berber, D. Tomanek, Phys. Rev. Lett. 101, 077401 (2008). [2] C-Y. Ruan, Y. Murooka, R.K. Raman, R.A. Murdick, R. Worhatch, A. Pell, Micro. Microanal. 15, 323 (2009). [3] R.K. Raman, Z. Tao, T-R. Han, C-Y. Ruan, Appl. Phys. Lett. 95, 181108 (2009). [4] This research was supported by US DOE under Award DE-FG0206ER46309. 